\title{
RHIC and LHC phenomena with an unified parton transport
}

\author{
loannis Bouras ${ }^{a}$, Andrej El ${ }^{a}$, Oliver Fochler ${ }^{a}$, Felix Reining ${ }^{a}$, Florian Senzel $^{a}$, Jan \\ Uphoff $^{a}$, Christian Wesp ${ }^{a}$, Zhe $\mathbf{X u}^{b}$ and Carsten Greiner ${ }^{* a}$ \\ ${ }^{a}$ Institut für Theoretische Physik, Johann Wolfgang Goethe-Universität \\ Max-von-Laue-Str. 1, D-60438 Frankfurt am Main, Germany \\ ${ }^{b}$ Department of Physics, Tsinghua University \\ Beijing 100084, China \\ E-Mail: carsten.greinereth.physik.uni-frankfurt.de
}

\begin{abstract}
We discuss recent applications of the partonic pQCD based cascade model BAMPS with focus on heavy-ion phenomeneology in hard and soft momentum range. The nuclear modification factor as well as elliptic flow are calculated in BAMPS for RHIC end LHC energies. These observables are also discussed within the same framework for charm and bottom quarks. Contributing to the recent jet-quenching investigations we present first preliminary results on application of jet reconstruction algorithms in BAMPS. Finally, collective effects induced by jets are investigated: we demonstrate the development of Mach cones in ideal matter as well in the highly viscous regime.
\end{abstract}

50th International Winter Meeting on Nuclear Physics, BORMIO2012

January 23-27, 2012

Bormio, Italy

\footnotetext{
*Speaker.
} 


\section{Introduction}

In collisions of heavy ions at ultrarelativistic energies at the Relativistic Heavy-Ion Collider (RHIC) and the Large Hadron Collider (LHC) a new state of matter, the quark-gluon plasma (QGP), has been created. Although the QGP is not available for direct observation, its properties can be deduced from the measurement of the produced hadrons in the final state.

The large values of the measured hadronic elliptic flow $v_{2}$ [1-3], which is the second coefficient of the Fourier series of the azimuthal particle multiplicity, suggests that equilibration of quarks and gluons occurs on a very short time scale $\leq 1 \mathrm{fm} / \mathrm{c}$. This also suggests that the shear viscosity over entropy density ratio $\eta / s$ of the QGP is very small, which means that the QGP behaves like a nearly perfect fluid. All these conclusions can be drawn from comparison of experimental results with hydrodynamic calculations. However, an understanding of the mechanism of fast thermalization can not be achieved in the scope of hydrodynamic models. The early pre-equilibrium dynamics of the QGP must be studied in the scope of the kinetic theory.

In contrast to the hydrodynamic approach, kinetic transport theory is a microscopic theory and thus allows to study processes of soft and hard processes simultaneously. This is in particular important for detailed understanding of further properties of the quark-gluonic medium, such as the suppression of jets and heavy-quarks. Suppression of jets, also known as jet quenching, is quantified by comparing the hadron multiplicities measured in heavy-ion collisions with appropriately scaled multiplicities from $p+p$ collisions [4-6]. In addition, very exciting jet-associated particle correlations were observed [7], which might be the result of a conical emission off propagating shock waves in form of Mach Cones. These Mach Cones might be induced by high-energy partons traversing the expanding medium [8]. Observations of these effects is consistent with the picture of a nearly perfect fluidity of the QGP.

The kinetic transport model BAMPS (Boltzmann Approach to Multiparton Scatterings) [9] has been developed to provide a unified description of dynamics of the early QGP stage of heavyion collisions (HIC) including perturbative QCD based elastic and inelastic processes. BAMPS has been applied to provide explanation of fast thermalization on a very short time scale $\leq 1 \mathrm{fm} / \mathrm{c}$ [10] as well as a small value of $\eta / s \approx 0.08-0.2$ for $\alpha_{s}=0.6-0.3$ [11, 12]. Furthermore recent calculations with BAMPS provide results on elliptic flow [13,14] and jet quenching [15] at RHIC energies, which is for the first time done in a consistent and fully pQCD-based microscopic transport model. In addition, BAMPS has been used in certain works as a reference for hydrodynamic calculations. This opens the possibility to study hydrodynamic phenomena for arbitrary viscosity.

In these proceedings we discuss application of BAMPS to describe a number of phenomena observed in the recent heavy-ion experiments. In Sec. 2 we introduce calculations of the nuclear modification factor $R_{A A}\left(p_{T}\right)$ for RHIC and LHC conditions. In Sec. 3 BAMPS results on elliptic flow and suppression of charm and bottom quarks for RHIC and LHC energies are introduced. In Sec. 4 for the first time in the framework of BAMPS we introduce preliminary results on application of jet reconstruction algorithms. Finally in Sec. 5 the formation and propagation of shock waves in form of Mach Cones are discussed for a wide range of viscosity to entropy density ratio $\eta / s$. 


\section{Nuclear modification factor and elliptic flow from partonic transport simulations}

As established in [13-15] the partonic medium in BAMPS simulations of ultra relativistic heavy ion collisions features a small ratio of the shear viscosity to the entropy, $\eta / s$, and develops a strong collectivity with an integrated $v_{2}$ that is in good agreement with experimental results over a large centrality range for a fixed coupling of $\alpha_{s}=0.3$ and a kinetic freeze-out energy density $\varepsilon_{c}=0.6^{\mathrm{GeV}} / \mathrm{fm}^{3}$. These parameters are used for all calculations that are presented in this section.

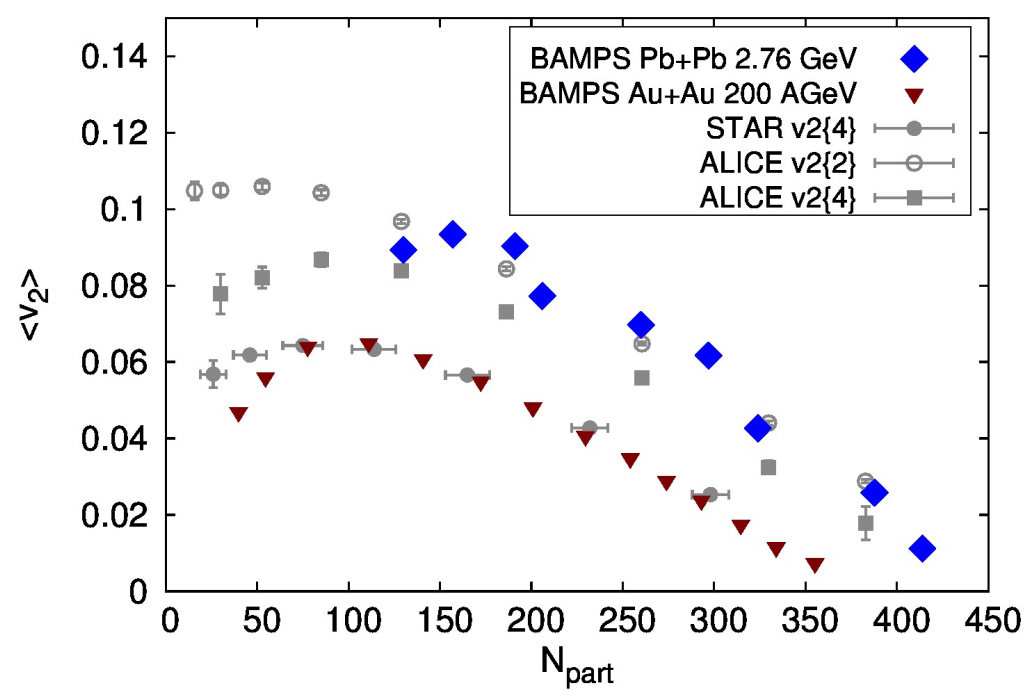

Figure 1: Integrated partonic $v_{2}$ from BAMPS as a function of $N_{\text {part }}$ for $\mathrm{Pb}+\mathrm{Pb}$ at $2.76 \mathrm{ATeV}(|y|<0.8)$ and $\mathrm{Au}+\mathrm{Au}$ at $200 \mathrm{AGeV}(|y|<0.5)$ compared to the measured $v_{2}$ of charged particles from ALICE [16] and from STAR [17].

Fig. 1 shows the integrated partonic $v_{2}$ as a function of centrality from simulations of $\mathrm{Au}+\mathrm{Au}$ at $2.76 \mathrm{~A} \mathrm{TeV}$ and of $\mathrm{Au}+\mathrm{Au}$ at $200 \mathrm{~A} \mathrm{GeV}$ compared to experimental data from the ALICE experiment [16] at LHC and from the STAR experiment [17] at RHIC. Using the same set of parameters $\left(\alpha_{s}=0.3, \varepsilon_{c}=0.6 \mathrm{GeV} / \mathrm{fm}^{3}\right)$ that have been fixed to the RHIC data $[13,14]$ also the integrated elliptic flow at LHC can be described over a large range in centrality. Accordingly the simulated differential $v_{2}$ of $\mathrm{Pb}+\mathrm{Pb}$ collisions at $\mathrm{LHC}$ energies shows no significant deviation from the $\mathrm{Au}+\mathrm{Au}$ results at $200 \mathrm{~A} \mathrm{GeV}$ in the low to intermediate $p_{T}$ region which is also in agreement with experimental findings [18].

One of the main virtues of the transport model BAMPS is that it allows for the investigation of different observables within a consistent framework and consequently also the nuclear modification factor, $R_{A A}$, is studied using the same parameters that provide a description of the elliptic flow $\left(\alpha_{s}=0.3, \varepsilon_{c}=0.6^{\mathrm{GeV}} / \mathrm{fm}^{3}\right)$. Fig. 2 shows the nuclear modification factor $R_{A A}$ obtained from BAMPS simulations of central, $0 \%-5 \%, \mathrm{~Pb}+\mathrm{Pb}$ collisions at $2.76 \mathrm{ATeV}$. The results are both shown on the partonic level for gluons and light quarks and on the hadronic level for neutral pions based on AKK fragmentation functions [19]. The suppression of high- $p_{T}$ particles in simulations with BAMPS is distinctly stronger than the experimentally observed suppression, which is also observed in simulations of $R_{A A}$ at RHIC energies [20]. Additionally the rise towards larger transverse 


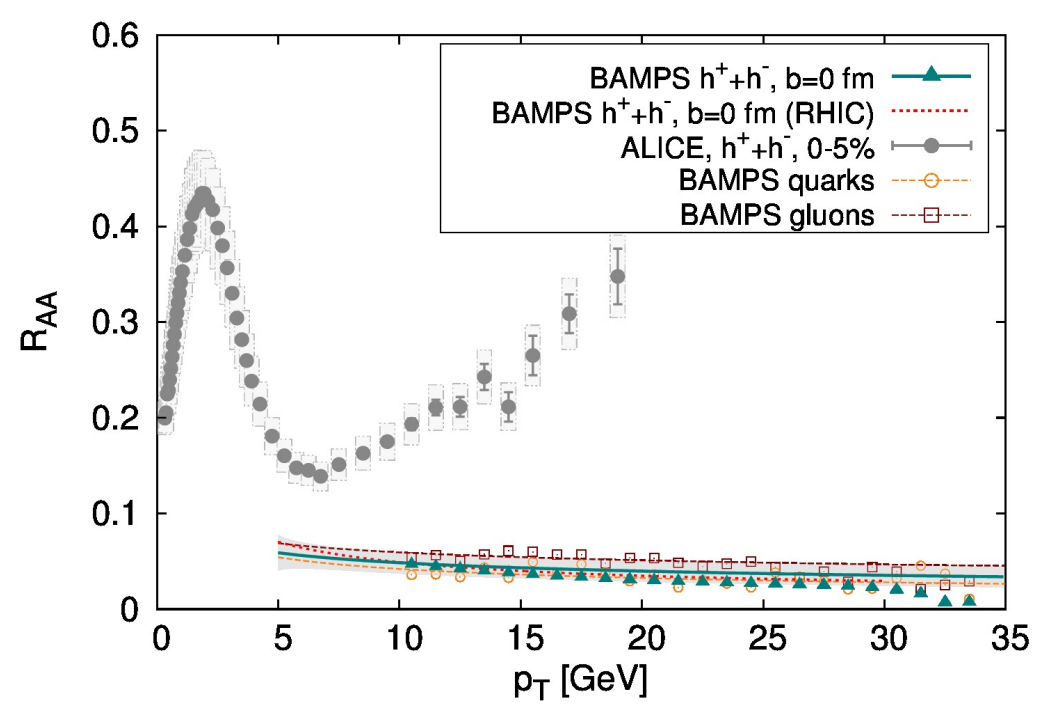

Figure 2: Nuclear modification factor $R_{A A}$ of charged hadrons, gluons and quarks from BAMPS simulations of $\mathrm{Pb}+\mathrm{Pb}$ at $b=0 \mathrm{fm}$ compared to results from ALICE for $0 \%$ to $5 \%$ central $\mathrm{Pb}+\mathrm{Pb}$ collisions [18]. Lines indicate $R_{A A}$ computed from fits to the simulated parton spectra, while symbols indicate $R_{A A}$ computed directly from the parton spectra as obtained from BAMPS. For comparison the $R_{A A}$ of charged hadrons from simulations of $\mathrm{Au}+\mathrm{Au}$ at $200 \mathrm{~A} \mathrm{GeV}$ and $b=0 \mathrm{fm}$ is also shown.

momenta that is present in the LHC data is not reproduced.

The strong quenching observed in BAMPS calculations is due to the energy loss in $2 \rightarrow 3$ interactions that include an effective implementation of the LPM effect [21] via a mean free pathdependent cutoff $[9,15]$. The strong quenching is then caused by [20] $a$ ) a strong energy loss that is caused by a complex interplay of the Gunion-Bertsch matrix element and the effective implementation of the LPM effect [20], $b$ ) a conversion of quark into gluon jets in $2 \rightarrow 3$ interactions and c) a small difference in the energy loss of gluons and quarks caused by the iterative computation of interaction rates required by the inclusion of the LPM cutoff.

Thus, while the collectivity of the medium can be well described within the current approach, the quenching of high- $p_{T}$ particles is overestimated. Future studies will therefore focus on the implementation of a running coupling for light quarks and gluons and also systematically explore the modeling of the LPM effect. These modifications are qualitatively expected to bring the results for the nuclear modification factors into better agreement with experimental data.

\section{Elliptic flow and suppression of heavy quarks}

Heavy quarks are a good probe to study the properties of the QGP. They are well calibrated in a sense that they are produced entirely in the early stage of the heavy ion collision due to their large mass [23] and are also tagged during hadronization due to flavor conservation. Whereas heavy quarks at RHIC can only be measured indirectly via heavy flavor electrons, at LHC for the first time it is possible to reconstruct $D$ mesons and, therefore, receive information only about charm quarks. 

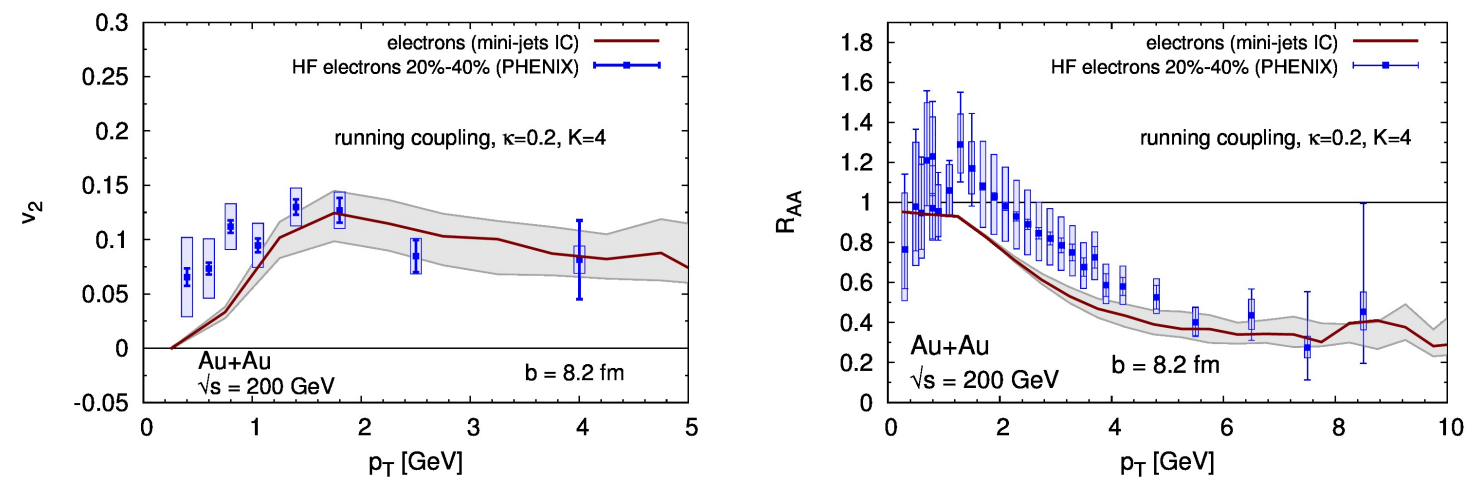

Figure 3: Elliptic flow $v_{2}$ (left) and nuclear modification factor $R_{A A}$ (right) of heavy flavor electrons for $\mathrm{Au}+\mathrm{Au}$ collisions at RHIC with an impact parameter of $b=8.2 \mathrm{fm}$ together with data [22]. The elastic cross section of $g Q \rightarrow g Q$ is multiplied with the factor $K=4$ to mimic the influence of radiative processes.
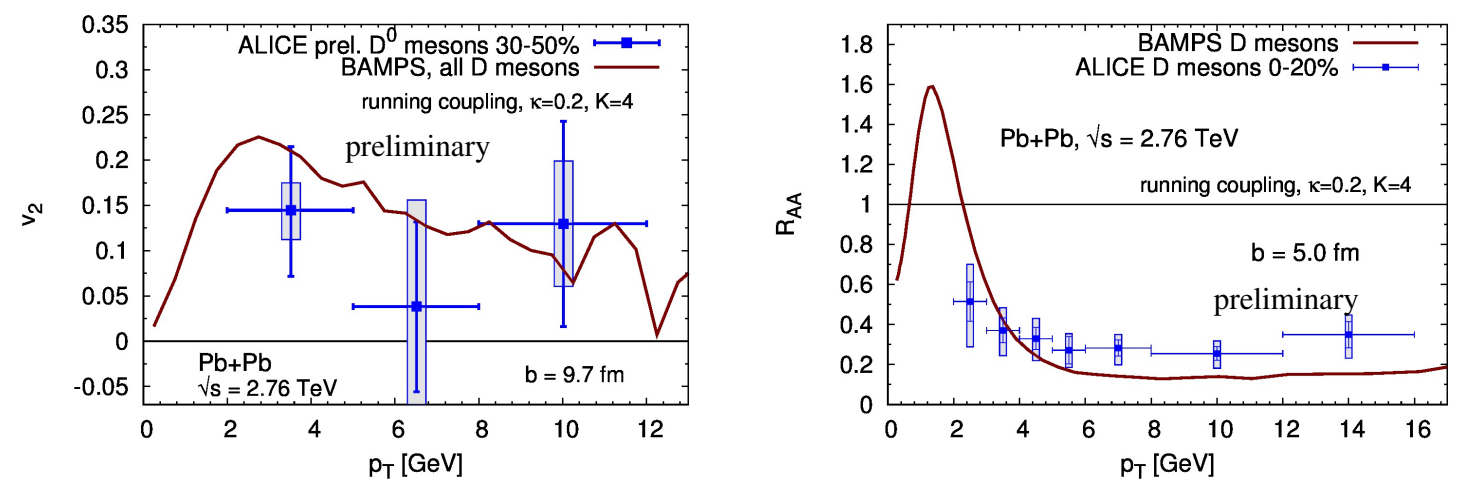

Figure 4: Preliminary BAMPS results on elliptic flow $v_{2}$ (left) and nuclear modification factor $R_{A A}$ (right) of $D$ mesons at $\mathrm{Pb}+\mathrm{Pb}$ collisions at LHC with an impact parameter $b$ together with data [24, 25]. The cross section of $g Q \rightarrow g Q$ is multiplied with the factor $K=4$.

The heavy flavor electron data from RHIC [22, 26, 27] and the heavy flavor electron, muon and $D$ meson data from LHC $[24,28]$ show that the suppression of heavy quarks is on the same order as for light quarks. From the theory perspective it was thought that radiative processes involving heavy quarks are suppressed due to the dead cone effect [29,30], which means that gluon radiation at small angles is suppressed and, therefore, the energy loss is smaller compared to light partons. Elliptic flow $v_{2}$ measurements of particles associated with open heavy flavor also show that heavy quarks interact strongly with the other particles of the medium. Whether these observations can be explained by collisional or radiative energy loss or other effects is currently in debate.

The elliptic flow $v_{2}$ and the nuclear modification factor $R_{A A}$ are important observables for heavy quarks. Although those particles are rare probes, both observables are experimentally accessible for fragmentation and decay products of heavy quarks such as $D$ mesons or heavy flavor electrons. The $R_{A A}$ reflects how much energy heavy quarks lose in the QGP. The $v_{2}$ is large if heavy quarks interact often with the medium and pick up its collective flow.

All the calculations for heavy quarks in this section are done with a running coupling and an 
improved Debye screening. The latter means that the screening mass of the $t$ channel of elastic scatterings is determined such that the energy loss matches the energy loss of a heavy quark calculated within the hard thermal loop approach. More information how this matching is done can be found in Ref. [31-34].

Detailed studies in BAMPS [33, 35-38] show that elastic energy loss of heavy quarks alone is not compatible with the experimental data at RHIC and LHC. However, elastic energy loss explains a significant portion of the overall suppression. If we employ a running coupling and improved Debye screening the experimental data for both $v_{2}$ and $R_{A A}$ for both RHIC and LHC can be explained if the elastic cross section is multiplied with the artificial factor $K=4$. This indicates that radiative energy loss should be three times larger than the elastic energy loss. However, this must be checked in a forthcoming study. First results on implementing radiative energy loss of heavy quarks in BAMPS look promising [35].

Figure 3 compares our results of the $v_{2}$ and $R_{A A}$ at RHIC to the heavy flavor electron data from Ref. [22]. The agreement with the experimental data is very good for both observables if one employs a factor $K=4$ for the elastic cross section to mimic the effect of radiative energy loss. At LHC for the first time it is possible to reconstruct $D$ mesons and, therefore, distinguish between charm and bottom quarks. In Fig. 4 our results on $D$ mesons is compared to data from ALICE. For the same parameters, that describe the RHIC data, a good agreement is also found at LHC. The suppression of $D$ mesons at LHC is slightly larger than the data. This can be due to a different relation between collisional and radiative processes at LHC compared to RHIC or due to the fact that we represent the rather large centrality class $0-20 \%$ by only one impact parameter. We note that muon data from charm and bottom quarks at forward rapidity is also well described for the same parameters [35].

\section{Jet reconstruction within BAMPS}

Another observable to determine the parton energy loss inside a heavy-ion medium is the reconstruction of full dijets. The initial hard scattering processes of the approaching nucleons lead to back-to-back parton pairs, which gain a high amount of virtuality during these scattering processes. In the subsequent evolution of partons, they try to decrease their virtuality by splitting processes like $\mathrm{q} \rightarrow \mathrm{qg}$ or $\mathrm{g} \rightarrow \mathrm{gg}$, which can be described by the DGLAP evolution equation [3941]. These fragmentation processes lead to particle showers with a broad angle and momentum distribution. In order to provide a description of the energy loss mechanism inside the created medium, jet reconstruction methods [42-44] are used. They combine single shower particles to a common "full jet" based on their distance $\Delta R=\sqrt{(\Delta y)^{2}+(\Delta \phi)^{2}}$ to the jet axis.

In $\mathrm{p}+\mathrm{p}$ collisions, where no medium creation is expected, these splitting processes already lead to an imbalance in the momenta of the reconstructed jets with the two highest transverse momenta. These jets are associated with the initial back-to-back parton pair and momentum asymmetry is caused by stochastic distributed vacuum splitting processes out of the considered jet cone. Experimental results [45-47] in $\sqrt{s_{N N}}=2.76 \mathrm{TeV} \mathrm{Pb}+\mathrm{Pb}$ collisions at the LHC showed an enhancement of this momentum imbalance in central HIC in comparison to $\mathrm{p}+\mathrm{p}$-collisions. As a 


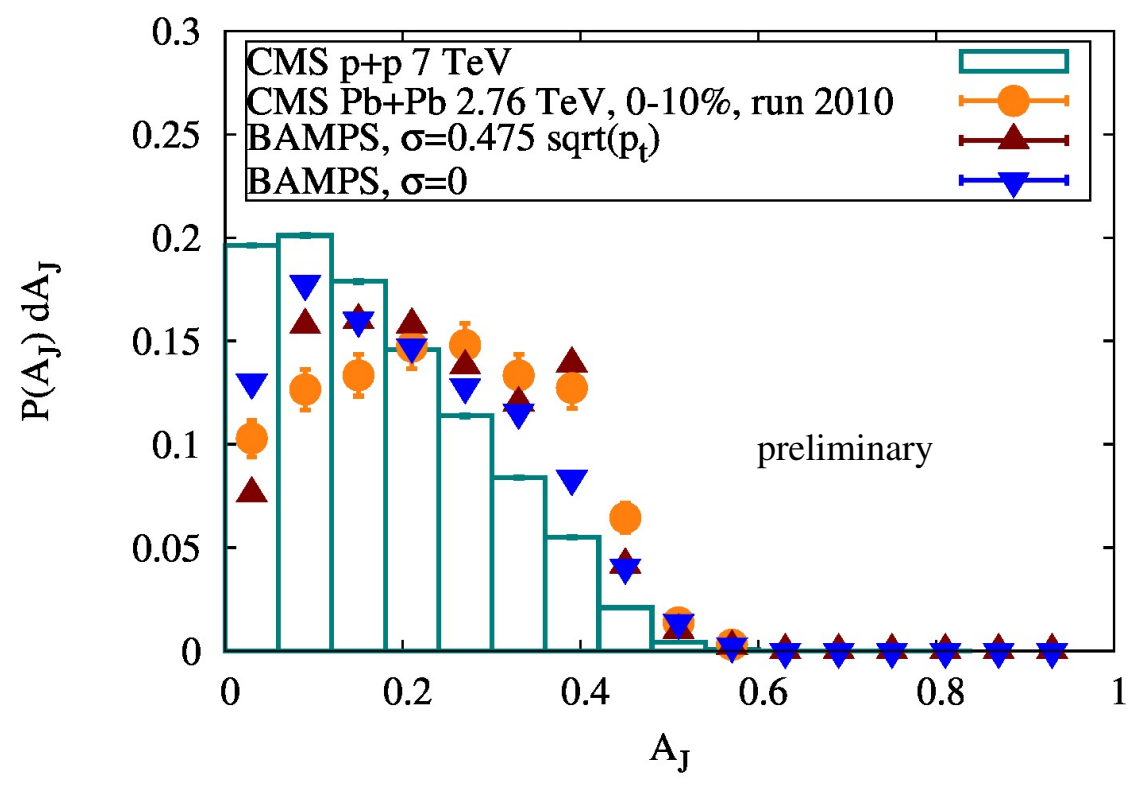

Figure 5: Momentum imbalance $A_{J}$ in central $\mathrm{Pb}+\mathrm{Pbcollisions}$ at the $\mathrm{LHC}$ with and without gaussian smearing for $\alpha_{s}=0.3$.

measure of this enhancement the momentum imbalance $A_{J}$,

$$
A_{J}=\frac{p_{\mathrm{t} ; \text { Leading }}-p_{\mathrm{t} ; \text { Subleading }}}{p_{\mathrm{t} ; \text { Leading }}+p_{\mathrm{t} ; \text { Subleading }}}
$$

is defined, where $p_{\mathrm{t} \text { :Leading }}\left(p_{\mathrm{t} \text {;Subleading }}\right)$ is the reconstructed transverse momentum of the jet with the highest (second highest) transverse momentum. The additional suppression of balanced events in HIC are supposed to be the result of different in-medium energy loss of the two partons within the created bulk medium, which is a consequence of a non-central spatial production point of the initial dijet pair.

In this section we present our first preliminary results on momentum imbalance simulated within the transport model BAMPS. For the initial momentum spectra of the partons we use a distribution sampled according to a parametrized parton distribution [48], starting at $p_{t ; 0}=100 \mathrm{GeV}$. Because BAMPS describes only scattering processes of particles on the mass-shell, it is necessary to model the initial splitting processes of the virtual partons properly for reproducing the findings in $p+p$ collisions. Therefore the shower routines of PYTHIA [49] are used to model the virtual splitting processes. Because the medium modification of the created parton showers is to be evaluated within the BAMPS framework, it is necessary to switch off hadronization processes and terminate the splitting processes within PYTHIA prematurely. Therefore, the standard PYTHIA global termination criterion in the virtuality $Q_{0}=1 \mathrm{GeV}$ is replaced by an energy-dependent minimum virtuality scale $Q_{0}=\sqrt{\frac{E_{\text {parton }}}{\tau}}$ depending on the individual parton energy and a global shower time $\tau$. Throughout this section the shower time is assumed as $\tau=0.2 \mathrm{fm}$. Calculations within a static medium showed that the energy loss of the reconstructed jets is, for realistic values of $\tau$, nearly independent of the used shower time. The initial spatial production points of the parton 


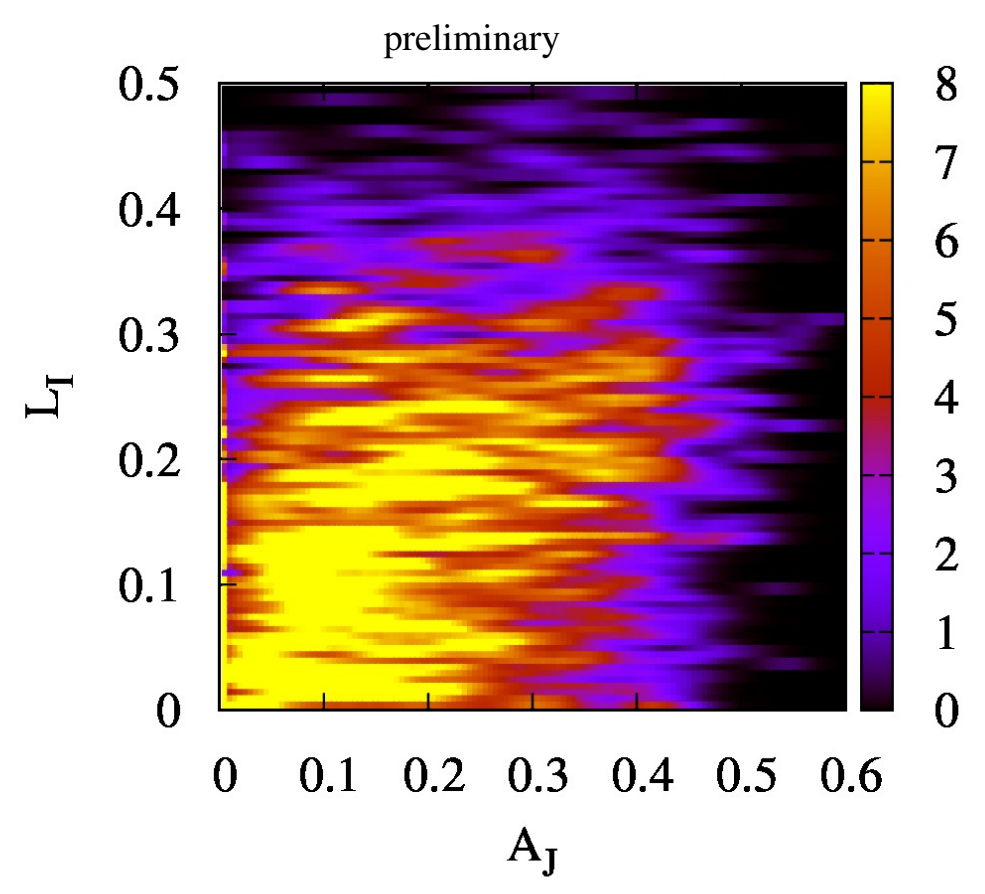

Figure 6: Distribution of length imbalance $L_{i}$ depending on $A_{J}$.

pairs are determined by a Glauber modelling of the initial nucleus-nucleus collisions based on a Woods-Saxon density profile.

The so created parton showers are evolved within an offline recorded BAMPS background event. At every timestep the shower particles can interact with medium particles which then become shower particle by their own. With this procedure it is possible to clearly discriminate between shower and background particles.

In the following we compare our simulations with the experimental data measured by CMS. All event trigger conditions by CMS $\left(p_{\mathrm{t} \text { :Leading }}>120 \mathrm{GeV}, p_{\mathrm{t} \text {; Subleading }}>50 \mathrm{GeV}, \Delta \phi>\frac{2 \pi}{3}\right.$ and $\left|\eta_{\text {jet }}\right|<2$ ) and an effective handling of the detector response and background fluctuations were used. For that an independent Gaussian smearing of the reconstructed jet momenta is applied. The width $\sigma$ is chosen in such a way that the smeared hadronic PYTHIA events without shower termination can reproduce the measured $\mathrm{p}+\mathrm{p}$ data by CMS [46].

Figure 5 shows the calculated $A_{J}$ distribution for central $\sqrt{s_{N N}}=2.76 \mathrm{TeV} \mathrm{Pb}+\mathrm{Pb}$ collisions $(0-10 \%$, which corresponds to a mean impact parameter $b=3.4 \mathrm{fm})$ with and without smearing of the reconstructed jet momenta. As one can see, already the "true" jet momenta lead to an increase in the momentum asymmetry, though it is insufficient to reproduce the measured experimental data at $A_{J}>0.3$. Therefore it can be assumed that the background fluctuations of the medium and the detector response play significant roles in explaining the strong imbalance in dijet momenta.

One advantage of simulations within a full $3+1 \mathrm{D}$ transport model is the availability of micro- 
scopic particle informations like space and momentum coordinates at every timestep. With this information it is possible to further investigate the processes leading to the observed momentum imbalance. The imbalance of the in-medium path lengths of the parton pair is studied introducing the length imbalance observable $L_{i}$

$$
L_{i}=\frac{L_{\mathrm{long}}-L_{\mathrm{short}}}{L_{\mathrm{long}}+L_{\mathrm{short}}}
$$

Lower values of $L_{i}$ correspond to equal paths of the partons inside the medium and thus more central production of the partons. The in-medium path lengths of the initial partons ( $\left.L_{\text {long }} / L_{\text {short }}\right)$ are determined by their spatial production point, their initial transverse momentum direction and their distance to the Wood-Saxon surface. Figure 6 shows the distribution of the lenght imbalance in bins of $A_{J}$. We observe that the length imbalance seems to be correlated to the underlying momentum asymmetry. One can state that the different transverse momenta of the reconstructed jets are mainly caused by the different in-medium path length of the two initial partons and hence a different energy loss. This suggests that there are events in which the parton pairs are produced in a more peripheral region so that one parton has to travel a longer distance through the medium than the other one, before leaving the collision zone.

We showed that the observation of a momentum imbalance by CMS is in agreement with simulated BAMPS events. Therefore we showed that the consideration of background fluctuations and detector responses plays significant role. This momentum imbalance is caused by a different in-medium path length of the two initial partons. Recent experimental results by CMS [47] with a lower cone radius $R=0.3$ and lower subleading jet trigger $p_{\text {t:Subleading Jet }}>30 \mathrm{GeV}$ show a broader and flatter $A_{J}$ distribution which can only be explained within BAMPS by usage of a higher cone radius $(R=0.5)$. This implies further investigations of the influence of bulk particles on the momentum of the reconstructed dijets. In addition, to understand the excess on energy loss of single hadrons simulated within BAMPS, it is highly necessary to study the relation between the momentum imbalance $A_{J}$ and the nuclear modification factor $R_{A A}$.

\section{Transition from ideal to viscous Mach cones in BAMPS}

Highly energetic partons propagating through the hot and dense QGP rapidly lose their energy and momentum as the energy is deposited in the medium. Measurements of two- and three-particle correlations in heavy-ion collisions show a complete suppression of the away-side jet, whereas for lower $p_{T}$ a double peak structure is observed in the two-particle correlation function [7]. One possible and promising origin of these structures is assumed to be the interaction of fast partons with the soft matter which generates collective motion of the medium in form of Mach cones. $[8,50]$.

For this purpose we investigate the propagation and formation of Mach cones in the microscopic transport model BAMPS (Boltzmann Approach of MultiParton Scatterings) [9] in the limit of vanishing mass and very small shear viscosity over entropy density ratio $\eta / s$ of the matter. Two different scenarios for the jet are used. In addition, by adjusting $\eta / s$, the influence of the viscosity on the profile of the Mach cone and the corresponding two-particle correlation is explored for the first time. The results presented are based on a recent publication [51]. 


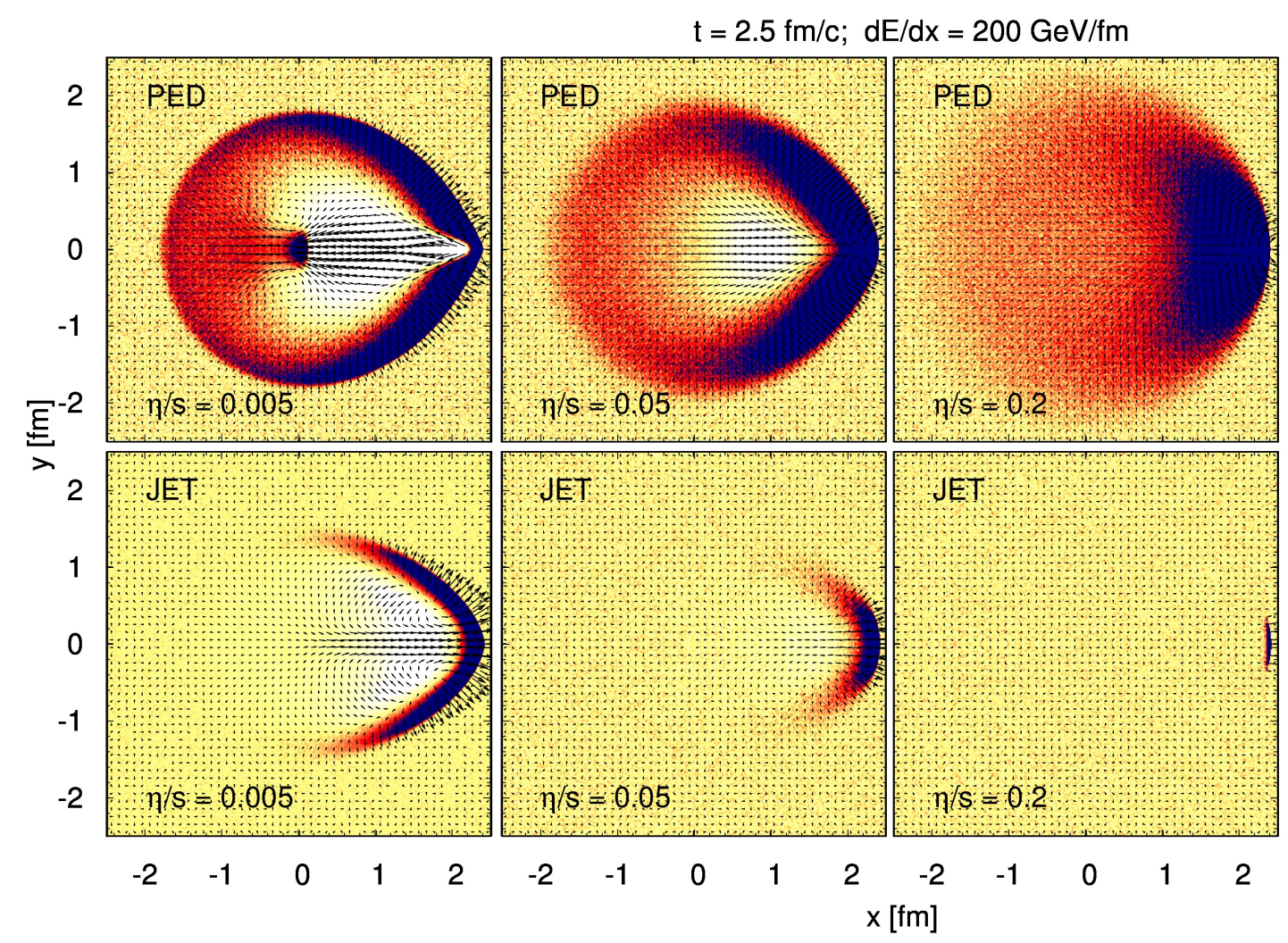

Figure 7: (Color online) Transition from ideal to viscous Mach cones. Shape of a Mach cone shown for different jet scenarios and different viscosity over entropy density ratios, $\eta / s=0.005,0.05$ and 0.5 . The energy deposition is $d E / d x=200 \mathrm{GeV} / \mathrm{fm}$. The upper panel shows the pure energy deposition scenario (PED); the lower panel shows the propagation of a highly energetic jet (JET) depositing energy and momentum in $x$-direction. Depicted are the LRF energy density within a specific range; as an overlay we show the velocity profile with a scaled arrow length. The results are a snapshot of the evolution at $t=2.5 \mathrm{fm} / \mathrm{c}$.

Shock waves are phenomena which have their origin in the collective motion of matter. In a simplified one-dimensional setup shock waves have already been studied within the framework of BAMPS for the perfect fluid limit [52, 53]. Furthermore BAMPS calculations have demonstrated that the shock profile is smeared out when viscosity is large. It was also found that a clear observation of the shock within the short time available in HIC requires a small viscosity.

In the following we study the evolution of "Mach cone"-like structures with different scenarios of the jet-medium interaction by using the parton cascade BAMPS. We focus on investigation of Mach cone evolution in absence of any other effects - i.e. we neglect such effects as initial fluctuations or expansion, which are however relevant in HIC. We use a static box with $T_{\text {med }}=400$ $\mathrm{MeV}$ and binary collisions with an isotropic cross section. Furthermore, we keep the mean free path $\lambda_{\text {mfp }}$ of the medium particles constant in all spatial cells by adjusting the cross section according to $\sigma=1 /\left(n \lambda_{\mathrm{mfp}}\right)$, where $n$ is the particle density. The related shear viscosity for isotropic binary collisions is given by $\eta=0.4 e \lambda_{\text {mfp }}$ [54].

The Mach Cones studied here are induced by two different sources. The first of them we refer to as the pure energy deposition scenario (PED) [55]. This is simulated by a moving source 


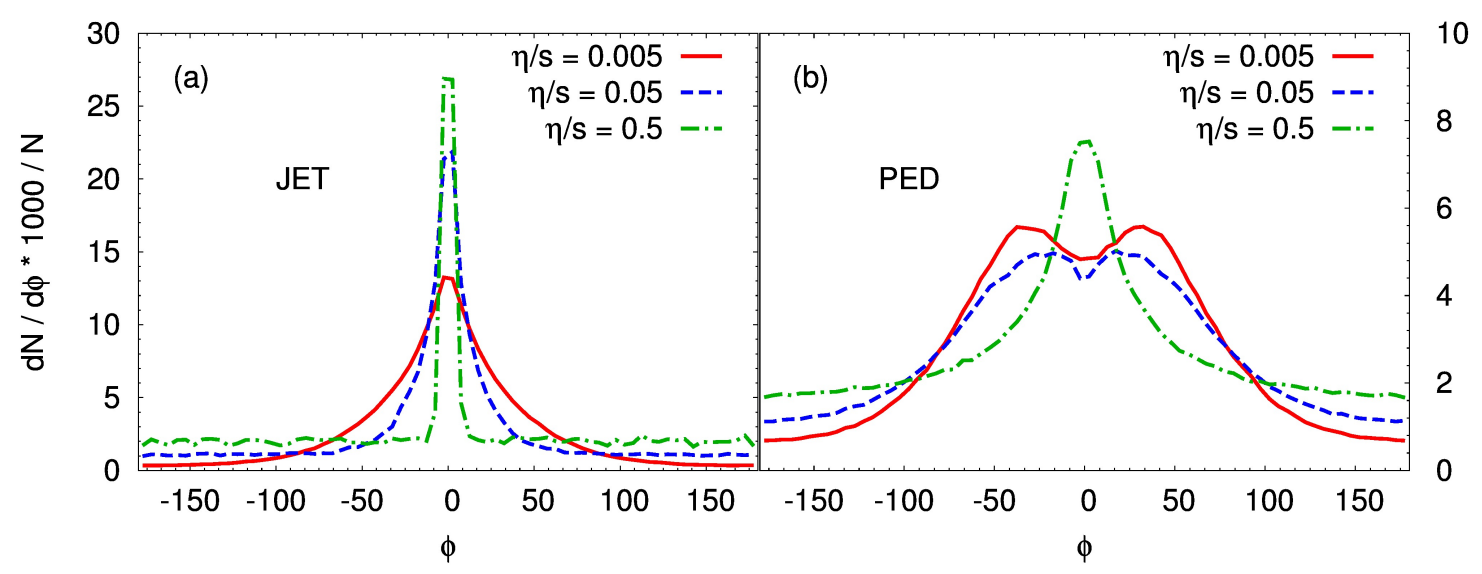

Figure 8: (Color online) Two-particle correlations $d N /(N d \phi)$ for different viscosities extracted from calculations shown in Fig. 7. The results are shown in the for the JET (a) and PED (b) scenario for $d E / d x=200$ $\mathrm{GeV} / \mathrm{fm}$.

depositing momentum end energy isotropically according to the thermal distribution $f(x, p)=$ $\exp (-E / T)$. The second source we refer to as JET. This is simulated by a highly massless particle (jet) which has only momentum in $x$-direction, i.e. $p_{\mathrm{x}}=E_{\text {jet }}$. After each timestep the energy of the jet particle is reset to its initial value. For both scenarios the sources are initialized at $t=0 \mathrm{fm} / \mathrm{c}$ at the position $x=-0.1 \mathrm{fm}$ and propagate in $x$-direction with $v_{\text {source }}=1$, i.e. with the speed of light.

In Fig. 7 we show the Mach Cone structure for both PED scenario (upper panel) and JET scenario (lower panel) with $\eta / s=0.005,0.05$ and 0.5 from left to right, respectively. We show a snapshot at $t=2.5 \mathrm{fm} / \mathrm{c}$. The energy deposition rate is fixed to $d E / d x=200 \mathrm{GeV} / \mathrm{fm}$. In both scenarios, PED and JET, for $\eta / s=0.005$ (left panel), we observe a conical structure, but with obvious differences. The PED case with the isotropic energy deposition induces a spherical shock into back region; this structure is missing in the JET scenario because of the high forward peaked momentum deposition. Another difference is that in the JET scenario a clearly visible head shock appears. This in turn is missing in the PED scenario. Furthermore a (anti)-diffusion wake is induced by the JET (PED) scenario.

Adjusting the shear viscosity over entropy density ratio $\eta / s=0.05-0.5$ we observe a smearing out of the Mach cone structure. For a sufficient high $\eta / s=0.5$ the conical structure in both scenarios disappears. This is true for shock fronts as well as for the (anti-) diffusion wake. The difference between the PED and the JET case is that as $\eta / s$ increases, in the PED scenario the resulting "Mach cone" solution covers approximately the same spatial region regardless of a value of $\eta / s$, while in the JET case the structure is concentrated more and more near the projectile as the viscosity increases.

In Fig. 8 we show the two-particle correlations extracted from BAMPS calculations of the Mach Cones shown in Fig. 7. For the JET scenario (a) and sufficiently small $\eta / s=0.005$ we observe only a peak in direction of the jet. The typical double peak structure, which has been proposed as a possible signature of the Mach cone in HIC, can only be observed for the PED scenario (b) and small $\eta / s$. However, the PED scenario has no correspondence in heavy-ion physics. We conclude that Mach cones can not be connected to double peak structures by any realistic picture 
of jets in HIC. In addition, finite values of the $\eta / s$ destroy any kind of Mach cone signatures.

\section{Acknowledgements}

The authors are grateful to the Center for the Scientific Computing (CSC) at Frankfurt for the computing resources. This work was supported by the Helmholtz International Center for FAIR within the framework of the LOEWE program launched by the State of Hesse.

\section{References}

[1] Stephen Scott Adler et al. Elliptic flow of identified hadrons in $\mathrm{Au}+\mathrm{Au}$ collisions at $\mathrm{s}(\mathrm{NN}) * *(1 / 2)=200-\mathrm{GeV}$. Phys. Rev. Lett., 91:182301, 2003.

[2] John Adams et al. Particle dependence of azimuthal anisotropy and nuclear modification of particle production at moderate $\mathrm{p}(\mathrm{T})$ in $\mathrm{Au}+\mathrm{Au}$ collisions at $\mathrm{s}(\mathrm{NN})^{* *}(1 / 2)=200-\mathrm{GeV}$. Phys. Rev. Lett., 92:052302, 2004.

[3] B. B. Back et al. Centrality and pseudorapidity dependence of elliptic flow for charged hadrons in $\mathrm{Au}+\mathrm{Au}$ collisions at $\mathrm{s}(\mathrm{NN})^{* *}(1 / 2)=200-\mathrm{GeV}$. Phys. Rev., C72:051901, 2005.

[4] John Adams et al. Transverse momentum and collision energy dependence of high $\mathrm{p}(\mathrm{T})$ hadron suppression in $\mathrm{Au}+\mathrm{Au}$ collisions at ultrarelativistic energies. Phys. Rev. Lett., 91:172302, 2003.

[5] C. Adler et al. Centrality dependence of high $\mathrm{p}(\mathrm{T})$ hadron suppression in $\mathrm{Au}+\mathrm{Au}$ collisions at $\mathrm{s}(\mathrm{NN}) * *(1 / 2)=130-\mathrm{GeV}$. Phys. Rev. Lett., 89:202301, 2002.

[6] K. Adcox et al. Suppression of hadrons with large transverse momentum in central $\mathrm{Au}+\mathrm{Au}$ collisions at $\mathrm{s}^{* *}(1 / 2)(\mathrm{N} \mathrm{N})=130-\mathrm{GeV}$. Phys. Rev. Lett., 88:022301, 2002.

[7] Fuqiang Wang. Measurement of jet modification at RHIC. J. Phys., G30:S1299-S1304, 2004.

[8] Horst Stoecker. Collective Flow signals the Quark Gluon Plasma. Nucl. Phys., A750:121147, 2005.

[9] Zhe Xu and Carsten Greiner. Thermalization of gluons in ultrarelativistic heavy ion collisions by including three-body interactions in a parton cascade. Phys. Rev., C71:064901, 2005.

[10] Andrej El, Zhe Xu, and Carsten Greiner. Thermalization of a color glass condensate and review of the 'Bottom-Up' scenario. Nucl. Phys., A806:287-304, 2008.

[11] Zhe Xu and Carsten Greiner. Shear viscosity in a gluon gas. Phys. Rev. Lett., 100:172301, 2008.

[12] Andrej El, Azwinndini Muronga, Zhe Xu, and Carsten Greiner. Shear viscosity and out of equilibrium dissipative hydrodynamics. Phys. Rev., C79:044914, 2009. 
[13] Zhe Xu, Carsten Greiner, and Horst Stocker. PQCD calculations of elliptic flow and shear viscosity at RHIC. Phys. Rev. Lett., 101:082302, 2008.

[14] Zhe Xu and Carsten Greiner. Elliptic flow of gluon matter in ultrarelativistic heavy- ion collisions. Phys. Rev., C79:014904, 2009.

[15] Oliver Fochler, Zhe $\mathrm{Xu}$, and Carsten Greiner. Towards a unified understanding of jetquenching and elliptic flow within perturbative QCD parton transport. Phys. Rev. Lett., 102:202301, 2009.

[16] K. Aamodt et al. Elliptic Flow of Charged Particles in $\mathrm{Pb}+\mathrm{Pb}$ Collisions at $\sqrt{s_{N N}}=2.76 \mathrm{TeV}$. Phys. Rev. Lett., 105:252302, 2010.

[17] John Adams et al. Azimuthal anisotropy in $\mathrm{Au}+\mathrm{Au}$ collisions at $\mathrm{s}(\mathrm{NN}) * *(1 / 2)=200-\mathrm{GeV}$. Phys. Rev., C72:014904, 2005.

[18] K. Aamodt et al. Suppression of charged particle production at large transverse momentum in central $\mathrm{Pb}+\mathrm{Pb}$ collisions at $\sqrt{s_{N N}}=2.76 \mathrm{TeV}$. Phys.Lett., B696:30-39, 2011.

[19] S. Albino, B.A. Kniehl, and G. Kramer. AKK update: Improvements from new theoretical input and experimental data. Nucl.Phys., B803:42-104, 2008.

[20] Oliver Fochler, Zhe $\mathrm{Xu}$, and Carsten Greiner. Energy loss in a partonic transport model including bremsstrahlung processes. 2010.

[21] L. D. Landau and I. Pomeranchuk. Limits of applicability of the theory of bremsstrahlung electrons and pair production at high energies. Dokl. Akad. Nauk Ser. Fiz., 92:535-536, 1953.

[22] A. Adare et al. Heavy quark production in $p+p$ and energy loss and flow of heavy quarks in au+au collisions at $\sqrt{s_{N} N}=200$ gev. Phys.Rev., C84:044905, 2011.

[23] Jan Uphoff, Oliver Fochler, Zhe Xu, and Carsten Greiner. Heavy quark production at RHIC and LHC within a partonic transport model. Phys. Rev., C82:044906, 2010.

[24] B. Abelev et al. Suppression of high transverse momentum D mesons in central $\mathrm{Pb}-\mathrm{Pb}$ collisions at $\sqrt{\left(s_{N N}\right)}=2.76 \mathrm{TeV} .2012$.

[25] Chiara Bianchin. Measurement of $\mathrm{D}^{0} \mathrm{v}_{2}$ in $\mathrm{Pb}-\mathrm{Pb}$ collisions at $\sqrt{\left(s_{N N}\right)}=2.76 \mathrm{TeV}$ with ALICE at the LHC. 2011.

[26] B. I. Abelev et al. Transverse momentum and centrality dependence of high-pt non-photonic electron suppression in Au+Au collisions at $\sqrt{s_{N N}}=200 \mathrm{GeV}$. Phys. Rev. Lett., 98:192301, 2007.

[27] A. Adare et al. Energy Loss and Flow of Heavy Quarks in Au+Au Collisions at $\sqrt{s_{N N}}=$ $200 \mathrm{GeV}$. Phys. Rev. Lett., 98:172301, 2007.

[28] S. Masciocchi. Investigation of charm and beauty production via semileptonic decays of heavy-flavour hadrons in $\mathrm{pp}$ at $7 \mathrm{TeV}$ and $\mathrm{Pb}-\mathrm{Pb}$ at $2.76 \mathrm{TeV}$ with ALICE. J.Phys.G, G38:124069, 2011. 
[29] Yuri L. Dokshitzer and D. E. Kharzeev. Heavy quark colorimetry of QCD matter. Phys. Lett., B519:199-206, 2001.

[30] Raktim Abir, Carsten Greiner, Mauricio Martinez, Munshi G. Mustafa, and Jan Uphoff. Soft gluon emission off a heavy quark revisited. Phys.Rev., D85:054012, 2012.

[31] P. B. Gossiaux and J. Aichelin. Towards an understanding of the RHIC single electron data. Phys. Rev., C78:014904, 2008.

[32] Andre Peshier. Turning on the Charm. 2008.

[33] Jan Uphoff, Oliver Fochler, Zhe Xu, and Carsten Greiner. Elliptic flow and energy loss of heavy quarks in ultra-relativistic heavy ion collisions. Phys.Rev., C84:024908, 2011.

[34] Alex Meistrenko, Andre Peshier, Jan Uphoff, and Carsten Greiner. Collisional energy loss of heavy quarks. 2012. 7 pages, 8 figures, 1 table, submitted to Physics Letters B.

[35] Jan Uphoff, Oliver Fochler, Zhe Xu, and Carsten Greiner. Open heavy flavor at RHIC and LHC in a partonic transport model. 2011.

[36] Oliver Fochler, Jan Uphoff, Zhe Xu, and Carsten Greiner. Jet quenching and elliptic flow at RHIC and LHC within a pQCD-based partonic transport model. J.Phys.G, G38:124152, 2011.

[37] Jan Uphoff, Oliver Fochler, Zhe Xu, and Carsten Greiner. Heavy quarks at RHIC and LHC within a partonic transport model. Nucl.Phys., A855:444-447, 2011.

[38] Jan Uphoff, Oliver Fochler, Zhe Xu, and Carsten Greiner. Production, elliptic flow and energy loss of heavy quarks in the quark-gluon plasma. 2010.

[39] V. N. Gribov and L. N. Lipatov. Deep inelastic e p scattering in perturbation theory. Sov. J. Nucl. Phys., 15:438-450, 1972.

[40] Yuri L. Dokshitzer. Calculation of the Structure Functions for Deep Inelastic Scattering and e+ e- Annihilation by Perturbation Theory in Quantum Chromodynamics. (In Russian). Sov. Phys. JETP, 46:641-653, 1977.

[41] Guido Altarelli and G. Parisi. Asymptotic Freedom in Parton Language. Nucl. Phys., B126:298, 1977.

[42] Gavin P Salam. Towards jetography. The European Physical Journal C, 67(3-4):637-686, 2010.

[43] Matteo Cacciari, Gavin P Salam, and Gregory Soyez. The anti- $k_{t}$ jet clustering algorithm. Journal of High Energy Physics, (04), 2008.

[44] Matteo Cacciari, Juan Rojo, Gavin P Salam, and Gregory Soyez. Jet Reconstruction in Heavy Ion Collisions. October 2010. 
[45] G. Aad et al. Observation of a Centrality-Dependent Dijet Asymmetry in Lead-Lead Collisions at $\sqrt{s}_{N N}=2.76 \mathrm{TeV}$ with the ATLAS Detector at the LHC. Physical Review Letters, 105(25):1-18, 2010.

[46] S. Chatrchyan, V. Khachatryan, A. Sirunyan, A. Tumasyan, W. Adam, et al. Observation and studies of jet quenching in $\mathrm{PbPb}$ collisions at $\sqrt{s}_{N N}=2.76 \mathrm{TeV}$. Physical Review C, 84(2), 2011.

[47] CMS Collaboration. Jet momentum dependence of jet quenching in $\mathrm{PbPb}$ collisions at $\operatorname{sqrt}(\mathrm{sNN})=2.76 \mathrm{TeV}$. Quantum, February 2012 .

[48] M. GlÃijck, E. Reya, and A. Vogt. Dynamical parton distributions of the proton and small x physics. Z. Phys., C67:433-448, 1995.

[49] TorbjÃuúrn SjÃústrand, Stephen Mrenna, and Peter Skands. PYTHIA 6.4 physics and manual. Journal of High Energy Physics, (05):026-026, 2006.

[50] Ioannis Bouras et al. Relativistic Shock Waves and Mach Cones in Viscous Gluon Matter. $J$. Phys. Conf. Ser., 230:012045, 2010.

[51] I. Bouras, A. El, O. Fochler, H. Niemi, Z. Xu, et al. Transition From Ideal To Viscous Mach Cones In A Kinetic Transport Approach. Phys.Lett., B710:641-646, 2012.

[52] I. Bouras et al. Relativistic shock waves in viscous gluon matter. Phys. Rev. Lett., 103:032301, 2009.

[53] I. Bouras et al. Investigation of shock waves in the relativistic Riemann problem: A comparison of viscous fluid dynamics to kinetic theory. Phys. Rev., C82:024910, 2010.

[54] S. R. de Groot et al. Relativistic Kinetic Theory: Principles and Applications. North Holland, Amsterdam, 1980.

[55] Barbara Betz, Jorge Noronha, Giorgio Torrieri, Miklos Gyulassy, Igor Mishustin, et al. Universality of the Diffusion Wake from Stopped and Punch-Through Jets in Heavy-Ion Collisions. Phys.Rev., C79:034902, 2009. 